\title{
Exponential Laws for Fractional Differences
}

\author{
By Godfrey L. Isaacs
}

\begin{abstract}
In Math. Comp., v. 28, 1974, pp. 185-202, Diaz and Osler gave the following (formal) definition for $\dot{\Delta}^{\alpha} f(z)$, the $\alpha$ th fractional difference of $f(z): \dot{\Delta}^{\alpha} f(z)=$ $\Sigma_{p=0}^{\infty} A_{p}^{-\alpha-1} f(z+\alpha-p)$. They derived formulas and applications involving this difference. They asked whether their differences satisfied an exponent law and what the relation was between their differences and others, such as $\Delta^{\alpha} f(z)=\Sigma_{p=0}^{\infty} A_{p}^{-\alpha-1} f(z+p)$. In this paper an exponent law for their differences is established and a relation found between the two differences mentioned above. Applications of these results are given.
\end{abstract}

1. Introduction. In $[2$, p. 186] Diaz and Osler give the following definition for $\dot{\Delta}^{\alpha} f(z)$, the $\alpha$ th fractional difference of $f(z)$ :

$$
\dot{\Delta}^{\alpha} f(z)=\sum_{p=0}^{\infty} A_{p}^{-\alpha-1} f(z+\alpha-p),
$$

where $A_{p}^{-\alpha-1}=\left(\begin{array}{c}p-\alpha-1 \\ p\end{array}\right)=(-1)^{p}\left(\begin{array}{l}\alpha \\ p\end{array}\right)$. (Note: in [2] $\dot{\Delta}^{\alpha}$ is written $\Delta^{\alpha}$.)

Since $A_{p}^{-\alpha-1}=O\left(p^{-\alpha-1}\right)$ as $p \rightarrow \infty$, the series is convergent for every $z$, if $f(t)=$ $O\left(t^{\alpha-\epsilon}\right)(\epsilon>0)$ as $|t| \rightarrow \infty$. Diaz and Osler show [2, p. 189], that if $z$ and $\alpha$ are fixed and if (in addition to the order condition above) $f(t)$ is analytic in a region $R$ containing the points $t=z+\alpha-p, p \geqslant 0$, then $\dot{\Delta}^{\alpha} f(z)$ may be put in the form of a line integral round a contour in $R$. They ask [2, p. 201] whether there is an exponent law for $\dot{\Delta}^{\alpha} f(z)$ of the form

$$
\dot{\Delta}^{r+s} f(z)=\dot{\Delta}^{r} \dot{\Delta}^{s} f(z)
$$

If $s_{n}=f(n)$, we obtain formally, for the sequence $s_{n}$,

$$
\dot{\Delta}^{\alpha} s_{n}=\sum_{p=0}^{\infty} A_{p}^{-\alpha-1} s_{n+\alpha-p} .
$$

If $\alpha=0,1,2, \ldots$, the series terminates at $p=\alpha$, and gives successive "backward differences," starting (at $\alpha=1$ ) with the difference $\dot{\Delta}^{1} s_{n}=s_{n+1}-s_{n}$.

2. An Exponent Law. In [3] the following definition for the $\alpha$ th fractional difference of a sequence $s_{n}$ was used:

$$
\Delta^{\alpha} s_{n}=\sum_{p=0}^{\infty} A_{p}^{-\alpha-1} s_{n+p},
$$

the series being supposed summable in some Cesàro sense. The definition is due to

Received January 2, 1979.

1980 Mathematics Subject Classification. Primary 33A70, 40A05; Secondary 65N10;

Key words and phrases. Fractional differences, successive differences, exponent law, summability of series. 
Chapman [1]. For $\alpha=0,1,2, \ldots$, the series terminates at $p=\alpha$ and we get successive "forward differences" starting (at $\alpha=1$ ) with the difference $\Delta^{1} s_{n}=s_{n}-s_{n+1}$. In fact, as is easily verified,

$$
\Delta^{\alpha} s_{n}=(-1)^{\alpha} \dot{\Delta}^{\alpha} s_{n} \quad(\alpha=0,1,2, \ldots) .
$$

If $\alpha$ is fractional, the formula (3) fails to make sense, since $\dot{\Delta}^{\alpha} s_{n}$ takes $s_{n}$ off its domain; further, (5) is no help since $(-1)^{\alpha}$ is neither real nor unique.

In [3, Theorem 1] the following exponent formula was obtained for the fractional differences (4):

$$
\Delta_{(C, \lambda)}^{r+s} s_{n}=\Delta_{(C, \lambda+s+\epsilon)}^{r} \Delta^{s} s_{n},
$$

where $\lambda \geqslant-1, \lambda+s \geqslant-1, r+s \neq 0,1,2, \ldots, \epsilon=0$ or $>0$ according to whether $s$ is or is not an integer, and (unfortunately) $r<0$ in the case $s \neq 0,1,2, \ldots$ Here it is assumed that the left side is summable $(C, \lambda)$. (The series giving $\Delta^{s} s_{n}$ is then automatically summable $(C, \mu)$, where $\mu \geqslant \max (\lambda+r,-1)$.)

Because of the failure to relate the definitions $\Delta^{\alpha} s_{n}$ and $\dot{\Delta}^{\alpha} s_{n}$ in the case $\alpha \neq$ $0,1,2, \ldots$, it did not seem likely that (6) could be of help in finding an exponent law of the type (2). However, if we write (2) out formally we obtain

(7) $\sum_{p=0}^{\infty} A_{p}^{-r-s-1} f(z+r+s-p)=\sum_{k=0}^{\infty} A_{k}^{-r-1} \sum_{m=0}^{\infty} A_{m}^{-s-1} f(z+r+s-k-m)$, and if we write (6) out, we get

$$
\sum_{p=0}^{\infty} A_{p}^{-r-s-1} s_{n+p}=\sum_{k=0}^{\infty} A_{k}^{-r-1} \sum_{m=0}^{\infty} A_{m}^{-s-1} s_{n+k+m} .
$$

We see that in (7) the same values of $f$ are used on both sides, namely $f(z+r+s-q)$, where $q=0,1,2, \ldots$, the jump from $f(z)$ to $f(z+s-m)$ occasioned by $\dot{\Delta}^{s}$ being overlaid by the subsequent jump due to $\dot{\Delta}^{r}$. Thus, if we put

$$
s_{q}=f(z+r+s-q) \quad(q=0,1,2, \ldots)
$$

in (8), with $n=0$, we obtain (7). We have thus obtained the following exponent law for Diaz and Osler's differences:

TheOREM 1. Let $\lambda \geqslant-1, \lambda+s \geqslant-1, r+s \neq 0,1,2, \ldots, \epsilon=0$ or $>0$ according as $s$ is integral or fractional, and $r<0$ if $s \neq 0,1,2, \ldots$ Then

$$
\dot{\Delta}_{(C, \lambda)}^{r+s} f(z)=\dot{\Delta}_{(C, \lambda+s+\epsilon)}^{r} \dot{\Delta}^{s} f(z),
$$

under the assumption that the left side is summable $(C, \lambda)$.

3. A "Converse" Exponent Law. In [3, Theorem 3] a "converse" result to (6) is given, which in its "convergence" form [3, Theorem $\left.3^{\prime}\right]$ is as follows:

$$
\Delta_{(C, 0)}^{r+s} s_{n}=\Delta_{(C, 0)}^{r} \Delta_{(C, 0)}^{s} s_{n}
$$

the two right side series being assumed convergent. Here (apart from the trivial cases $r=0$ or $s=0) r$ and $s$ must be in the first or fourth quadrant or inside the open triangles with vertices $(0, k),(0, k+1),(-1, k+1), k=0,1,2, \ldots$ From this we obtain the corresponding formula for Diaz and Osler's differences: 
THEOREM 2. If $r$ and $s$ are in the set $S$ just described,

$$
\dot{\Delta}_{(C, 0)}^{r+s} f(z)=\dot{\Delta}_{(C, 0)}^{r} \dot{\Delta}_{(C, 0)}^{s} f(z),
$$

the two right side series being supposed convergent.

The last formula is useful in extending known results of Diaz and Osler. In [2, Table 2.1], they give $\dot{\Delta}^{\alpha} f(z)$ for some special functions $f(z)$. In each case it can be seen that the two series on the right side of (12) are convergent for the value of $\alpha(=s)$ given, and for $r$ and $s$ in the set $S$; hence, we know that the $r$ th difference of the expression $\dot{\Delta}^{\alpha} f(z)(\alpha=s)$ given in the table is just the difference $\dot{\Delta}^{r+s}$ of the function $f(z)$. In short, the functions $f(z)$ given in the table all satisfy the exponent law (12) with suitable restrictions on $r$ and $s$.

4. An Example. As an example of the above, let

$$
f(z)=z^{(p)}=\frac{\Gamma(z+1)}{\Gamma(z+1-p)} .
$$

Then by [2, Table 2.1$]$, with $s$ for $\alpha$,

$$
\dot{\Delta}^{s} f(z)=\frac{\sin (\pi z) \Gamma(s-p) z^{(p-s)}}{\sin (\pi(z+s)) \Gamma(-p)}
$$

for $s>p$. (It is assumed that both $z^{(p)}$ and $\dot{\Delta}^{s} f(z)$ are defined by continuity at points of removable singularity, and that $z, p, s$ are chosen so as to avoid points of unremovable singularity in either of them; thus if, in $z^{(p)}, z$ is a negative integer, so must $z-p$ be, and if, in $\dot{\Delta}^{s} f(z), z+s$ is an integer, then $p$ is 0 or a positive integer.) Now

$$
\dot{\Delta}^{r} \dot{\Delta}^{s} f(z)=\sum_{k=0}^{\infty} A_{k}^{-r-1}\left(\dot{\Delta}^{s} f(z+r-k)\right) .
$$

Replacing $z$ by $z+r-k$ in (13), we see that $\dot{\Delta}^{s} f(z+r-k)$ is $O(|z|+k)^{p-s}$ as $k \rightarrow \infty$. Hence, since $A_{k}^{-r-1}$ is $O\left(k^{-r-1}\right)$, the series in (14) converges if $r+s>p$. (To avoid unremovable singularities in the terms of the series of (14) we see that if, for any $k, z+r-k+s$ is an integer, then we must take $p=0$ or a positive integer; and it is gratifying to see that this happens if and only if, whenever $z+r+s$ is an integer, then $p=0,1,2, \ldots$, which is the criterion that $\dot{\Delta}^{r+s} f(z)$ has no unremovable singularity.)

Hence the equality in (12) is true for $f(z)=z^{(p)}$ with $s>p, r+s>p$, and $r, s$ in the set $S$ (and, of course, $p=0,1,2, \ldots$, if $z+r+s$ happens to be an integer). In particular, if $p \geqslant 0$ and $z+r+s$ is nonintegral, (12) is true if $s>p$ and $r>0$, a useful case. The arguments for the other functions $f(z)$ of Table 2.1 are similar.

5. Relation Between $\Delta^{\alpha}$ and $\dot{\Delta}^{\alpha}$. Although there is no extension of the DiazOsler differences (1) to sequences, for $\alpha$ fractional, there is an immediate extension of the differences (4) to functions $f(z)$ :

$$
\Delta^{\alpha} f(z)=\sum_{p=0}^{\infty} A_{p}^{-\alpha-1} f(z+p) .
$$

Diaz and Osler ask [2, p. 201] whether there is a relation between (1) and other dif- 
ferences. Now for $\alpha=0,1,2, \ldots$, we can replace $\infty$ in (1) by $\alpha$ and then replace $p$ by $\alpha-p$. This shows that by (15),

$$
\Delta^{\alpha} f(z)=(-1)^{\alpha} \dot{\Delta}^{\alpha} f(z) .
$$

But as with $s_{n}$ in (5), this has no meaning for $\alpha$ fractional.

Let us write for given fixed $z$ and $\alpha$,

Then it is easy to verify:

$$
g(u)=f(2 z+\alpha-u) .
$$

THEOREM 3. If the series for either side converges, then

where $g(u)$ is given by (17).

$$
\Delta^{\alpha} f(z)=\left(\dot{\Delta}^{\alpha} g(u)\right)_{u=z}
$$

This enables us to calculate $\Delta^{\alpha} f(z)$ from known differences of the $\dot{\Delta}$ type. For example, let

$$
f(z)=z^{(p)}=\frac{\Gamma(z+1)}{\Gamma(z-p+1)} .
$$

Then

$$
g(u)=f(2 z+\alpha-u)=(2 z+\alpha-u)^{(p)}=\frac{\Gamma(2 z+\alpha+1-u)}{\Gamma(2 z+\alpha+1-p-u)}=\frac{\Gamma(A-u)}{\Gamma(B-u)},
$$

say. Thus by $[2$, Table $2.1, \# 4]$,

$$
\begin{aligned}
\Delta^{\alpha} f(z)=\left(\dot{\Delta}^{\alpha} g(u)\right)_{u=z} & =\left(\frac{\Gamma(B-A+\alpha) \Gamma(A-\alpha-u)}{\Gamma(B-A) \Gamma(B-u)}\right)_{u=z} \quad(B-A>-\alpha) \\
& =\left(\frac{\Gamma(-p+\alpha) \Gamma(2 z+1-u)}{\Gamma(-p) \Gamma(2 z+\alpha+1-p-u)}\right)_{u=z} \\
& =\frac{\Gamma(\alpha-p) \Gamma(z+1)}{\Gamma(-p) \Gamma(z+\alpha+1-p)} \\
& =\frac{\Gamma(\alpha-p)}{\Gamma(-p)} z^{(p-\alpha)}
\end{aligned}
$$

for $\alpha>p$.

By (13) this gives

$$
\Delta^{\alpha} f(z)=\frac{\sin (\pi(z+\alpha))}{\sin (\pi z)} \dot{\Delta}^{\alpha} f(z)
$$

when $\alpha>p$, which is a direct extension of (16) to fractional values of $\alpha$.

\section{Department of Mathematics}

Herbert H. Lehman College, City University of New York

Bronx, New York 10468

1. S. CHAPMAN, "On non-integral orders of summability of series and integrals," Proc. London Math. Soc. (2), v. 9, 1911 , pp. 369-409.

2. J. B. DIAZ \& T. J. OSLER, "Differences of fractional order," Math. Comp., v. 28, 1974, pp. 185-202.

3. G. L. ISAACS, "An iteration formula for fractional differences," Proc. London Math. Soc. (3), v. 13, 1963, pp. 430-460. 